\title{
FUZZY BARRELS ON LOCALLY CONVEX FUZZY TOPOLOGICAL VECTOR SPACES
}

\author{
B. DARABY, N. KHOSRAVI, AND A. RAHIMI
}

Received 16 January, 2020

\begin{abstract}
In this paper, we introduce the concept of fuzzy barrels on locally convex fuzzy topological vector spaces. We present some characterizations of the fuzzy locally convex spaces, which are fuzzy barrelled. Using fuzzy barrels, we prove that the same fuzzy sets are bounded in any fuzzy topology of dual pair. Finally, we prove the Banach-Steinhaus theorem for the fuzzy topological vector spaces.
\end{abstract}

2010 Mathematics Subject Classification: 03E72; 54A40

Keywords: polar fuzzy sets, fuzzy barrel, fuzzy barrelled spaces

\section{INTRODUCTION}

In [13], the concept of fuzzy topological vector space was introduced by Katsaras and Liu. In [11], Katsaras changed the definition of fuzzy topological vector space and he considered the linear fuzzy topology on the scalar field $\mathbb{K}(\mathbb{R}$ or $\mathbb{C}$ ), which consists of all lower semi-continuous functions from $\mathbb{K}$ into $I=[0,1]$. At the first, the idea of fuzzy norm on linear spaces introduced by Katsaras. After then, in different approach many authors like Cheng and Mordeson [2], Felbin [10], Bag and Samanta [1], etc. introduced the notion of fuzzy normed linear space. Later, Xiao and Zhu [16], Fang [9], Daraby et. al. [5, 6], redefined the notion of Felbin's [10] definition of fuzzy norm. Das [7] introduced a fuzzy topology generated by fuzzy norm and studied some properties of this topology.

The concept of weak linear fuzzy topology on a fuzzy topological vector space as a generalization of usual weak topology was studied in [4]. Also, in [4] the authors have proved that this fuzzy topology consists of all weakly lower semi-continuous fuzzy sets on a given vector space when $\mathbb{K}$ endowed with its usual fuzzy topology. In [3], the concept of polar fuzzy sets on fuzzy dual spaces was studied. Also, in [3] the polar linear fuzzy topologies on fuzzy dual spaces have introduced by the help of polar fuzzy sets and a Mackey-Arens type theorem on fuzzy topological vector spaces has investigated. 
One of the most powerful theorems of functional analysis is the Banach-Steinhaus theorem. This theorem asserts that the set of continuous linear mapping that is pointwise bounded is bounded uniformly on barrelled spaces. This shows the importance of the barrelled spaces in functional analysis. In this paper we are going to study the fuzzy barrelled spaces.

\section{Preliminaries}

Let $X$ be a non-empty set. A fuzzy set in $X$ is an element of the set $I^{X}$ of all functions from $X$ into $I$.

Definition 1 ([7]). Let $X$ and $Y$ be any two non-empty sets, $f: X \rightarrow Y$ be a mapping and $\mu$ be a fuzzy subset of $X$. Then $f(\mu)$ is a fuzzy subset of $Y$ defined by

$$
f(\mu)(y)= \begin{cases}\sup _{x \in f^{-1}(y)} \mu(x) & f^{-1}(y) \neq \varnothing, \\ 0 & \text { else, }\end{cases}
$$

for all $y \in Y$, where $f^{-1}(y)=\{x: f(x)=y\}$. If $\eta$ is a fuzzy subset of $Y$, then the fuzzy subset $f^{-1}(\eta)$ of $X$ is defined by $f^{-1}(\eta)(x)=\eta(f(x))$ for all $x \in X$.

Definition 2 ([11]). A fuzzy topology on a set $X$ is a subset $\tau_{f}$ of $I^{X}$ satisfying the following conditions:

(i) $\tau_{f}$ contains every constant fuzzy set in $X$,

(ii) if $\mu_{1}, \mu_{2} \in \tau_{f}$, then $\mu_{1} \wedge \mu_{2} \in \tau_{f}$,

(iii) if $\mu_{i} \in \tau_{f}$ for each $i \in A$, then $\sup _{i \in A} \mu_{i} \in \tau_{f}$.

The pair $\left(X, \tau_{f}\right)$ is called a fuzzy topological space.

The elements of $\tau_{f}$ are called fuzzy open sets in $X$.

Definition 3 ([7]). A fuzzy topological space $\left(X, \tau_{f}\right)$ is said to be fuzzy Hausdorff if for $x, y \in X$ and $x \neq y$ there exist $\eta, \beta \in \tau_{f}$ with $\eta(x)=\beta(y)=1$ and $\eta \wedge \beta=0$.

A mapping $f$ from a fuzzy topological space $X$ into a fuzzy topological space $Y$ is called fuzzy continuous if $f^{-1}(\mu)$ is fuzzy open in $X$ for each fuzzy open set $\mu$ in $Y$. Suppose $X$ is a fuzzy topological space and $x \in X$. A fuzzy set $\mu$ in $X$ is called a neighborhood of $x \in X$ if there is a fuzzy open set $\eta$ with $\eta \leq \mu$ and $\eta(x)=\mu(x)>0$. Warren [15] has proved that a fuzzy set $\mu$ in $X$ is fuzzy open if and only if $\mu$ is a neighborhood of $x$ for each $x \in X$ with $\mu(x)>0$. The collection $O$ of fuzzy sets is called a fuzzy open covering of $\mu$ when $\mu \leq \sup _{v \in O} v$. The fuzzy set $\mu$ is called fuzzy compact if any fuzzy open covering of $\mu$ has finite subcovering.

Definition 4 ([7]). If $\mu_{1}$ and $\mu_{2}$ are two fuzzy subsets of a vector space $E$, then the fuzzy set $\mu_{1}+\mu_{2}$ is defined by

$$
\left(\mu_{1}+\mu_{2}\right)(x)=\sup _{x=x_{1}+x_{2}}\left(\mu_{1}\left(x_{1}\right) \wedge \mu_{2}\left(x_{2}\right)\right) .
$$


If $t \in \mathbb{K}$, we define the fuzzy sets $\mu_{1} \times \mu_{2}$ and $t \mu$ as follows:

$$
\left(\mu_{1} \times \mu_{2}\right)\left(x_{1}, x_{2}\right)=\min \left\{\mu_{1}\left(x_{1}\right), \mu_{2}\left(x_{2}\right)\right\}
$$

and

(i) for $t \neq 0,(t \mu)(x)=\mu\left(\frac{x}{t}\right)$ for all $x \in E$,

(ii) for $t=0,(t \mu)(x)= \begin{cases}0 & x \neq 0, \\ \sup _{y \in E} \mu(y) & x=0 .\end{cases}$

A fuzzy set $\mu$ in vector space $E$ is called balanced if $t \mu \leq \mu$ for each scalar $t$ with $|t| \leq 1$. As, it has been shown in [13], $\mu$ is balanced if and only if $\mu(t x) \geq \mu(x)$ for each $x \in E$ and each scalar $t$ with $|t| \leq 1$. Also, when $\mu$ is balanced, we have $\mu(0) \geq \mu(x)$ for each $x \in E$. The fuzzy set $\mu$ is called absorbing if and only if $\sup _{t>0} t \mu=1$. Then a fuzzy set $\mu$ is absorbing whenever $\mu(0)=1$. We shall say that the fuzzy set $\mu$ is convex if and only if for all $t \in I, t \mu+(1-t) \mu \leq \mu$, [11]. Also a fuzzy set $\mu$ is called absolutely convex if it is balanced and convex.

Definition 5 ([8]). A fuzzy topology $\tau_{f}$ on a vector space $E$ is said to be a fuzzy linear topology, if the mappings

$$
\begin{aligned}
& f: E \times E \rightarrow E,(x, y) \rightarrow x+y, \\
& g: \mathbb{K} \times E \rightarrow E,(t, x) \rightarrow t x,
\end{aligned}
$$

are continuous when $\mathbb{K}$ is equipped with the fuzzy topology induced by the usual topology, $E \times E$ and $\mathbb{K} \times E$ are the corresponding product fuzzy topologies. A vector space $E$ with a fuzzy linear topology $\tau_{f}$, denoted by the pair $\left(E, \tau_{f}\right)$, is called fuzzy topological vector space (abbreviated to FTVS).

Definition 6 ([11]). Let $\left(E, \tau_{f}\right)$ be a fuzzy topological vector space, the collection $v \subset \tau_{f}$ of neighborhoods of zero is a local base whenever for each neighborhood $\mu$ of zero and each $\theta \in(0, \mu(0))$ there is $\gamma \in v$ such that $\gamma \leq \mu$ and $\gamma(0)>\theta$.

Definition 7 ([12]). A fuzzy seminorm on $E$ is a fuzzy set $\mu$ in $E$ which is absolutely convex and absorbing.

We say that a fuzzy set $\mu$, in a vector space $E$, absorbs the fuzzy set $\eta$ if $\mu(0)>0$ and for every $\theta<\mu(0)$ there exists $t>0$ such that $\theta \wedge(t \eta) \leq \mu$. A fuzzy set $\mu$ in a fuzzy linear space $E$ is called bounded if it is absorbed by every neighborhood of zero.

The Katsaras norm was defined as follows:

Definition 8 ([12]). A fuzzy norm on vector space $E$ is an absolutely convex and absorbing fuzzy set $\rho$ with $\inf _{t>0}(t \rho)(x)=0$, for $x \neq 0$.

Definition 9 ([12]). A fuzzy topological vector space $\left(E, \tau_{f}\right)$ is called locally convex if it has a neighborhood base at zero consisting of convex fuzzy sets. 
Let $(E, \tau)$ be a topological vector space. The collection of all lower semicontinuous functions from $E$ in to $[0,1]$ is a fuzzy linear topology on $E$ denoted by $w(\tau)$.

Proposition 1 ([3]). Let $\left(E, \tau_{f}\right)$ be a fuzzy topological vector space. Then there is a linear topology $\tau$ on $E$ such that $\tau_{f}=w(\tau)$.

In [14], the concept of dual pair defined as follows:

We call $\left(E, E^{\prime}\right)$ a dual pair, whenever $E$ and $E^{\prime}$ are two vector spaces over the same $\mathbb{K}$ scaler field and $\left\langle x, x^{\prime}\right\rangle$ is a bilinear form on $E$ and $E^{\prime}$ satisfying the following conditions:

(D) For each $x \neq 0$ in $E$, there is $x^{\prime} \in E^{\prime}$ such that $\left\langle x, x^{\prime}\right\rangle \neq 0$.

$\left(D^{\prime}\right)$ For each $x^{\prime} \neq 0$ in $E^{\prime}$ there is $x \in E$ such that $\left\langle x, x^{\prime}\right\rangle \neq 0$.

Let $\left(E, E^{\prime}\right)$ be a dual pair. We denote by $\sigma_{f}\left(E, E^{\prime}\right)$, the weakest linear fuzzy topology on $E$ which makes all $x^{\prime} \in E^{\prime}$ fuzzy continuous as linear functionals from $E$ into $(\mathbb{K}, w(\tau))$, where $\tau$ is the usual topology on $\mathbb{K}$. We call $\sigma_{f}\left(E, E^{\prime}\right)$, the weak fuzzy topology (see [4]).

Lemma 1 ([4]). Let $\left(E, E^{\prime}\right)$ be a dual pair. If we consider the usual fuzzy topology $\omega(\tau)$ on $\mathbb{K}$, then

$$
\sigma_{f}\left(E, E^{\prime}\right)=\omega\left(\sigma\left(E, E^{\prime}\right)\right)
$$

where $\sigma\left(E, E^{\prime}\right)$ usual weak topology on $E$.

Let $E$ be a vector space and $\mu \in I^{E}$. The $\theta$-level set of $\mu$ is defined by

$$
[\mu]_{\theta}=\{x \in E: \mu(x) \geq \theta\},
$$

where $0<\theta \leq 1$. It is clear that for $\theta_{1}, \theta_{2} \in(0,1]$ with $\theta_{1}<\theta_{2}$ we have $[\mu]_{\theta_{1}} \supseteq[\mu]_{\theta_{2}}$. Therefore $\left\{[\mu]_{\theta}: \theta \in(0,1]\right\}$ is a decreasing collection of subsets of $E$.

Proposition 2 ([3]). Let $\left(E, \tau_{f}\right)$ be a fuzzy topological vector space and $\tau_{f}=w(\tau)$. Then the following hold.

(a) The fuzzy set $\mu \in I^{E}$ is fuzzy compact if and only if $[\mu]_{\theta}$ is compact in $(E, \tau)$ for each $0<\theta \leq 1$,

(b) The fuzzy set $\mu \in I^{E}$ is fuzzy closed if and only if $[\mu]_{\theta}$ is closed in $(E, \tau)$ for each $0<\theta \leq 1$,

(c) The fuzzy set $\mu \in I^{E}$ is fuzzy absolutely convex if and only if $[\mu]_{\theta}$ is absolutely convex in $(E, \tau)$ for each $0<\theta \leq 1$.

\section{FUZZY BARRELLED SPACES}

The concept of polar of fuzzy sets was introduced in [3] as follows:

Let $\left(E, E^{\prime}\right)$ be a dual pair. For the non-empty subset $A$ of $E$, its polar $A^{\circ}$ defined by:

$$
A^{\circ}=\left\{x^{\prime} \in E^{\prime}: \sup _{x \in A}\left|\left\langle x, x^{\prime}\right\rangle\right| \leq 1\right\} .
$$


Definition 10 ([3]). Let $\left(E, E^{\prime}\right)$ be a dual pair, $\mu \in I^{E}$. For $x^{\prime} \in E^{\prime}$ and $\mu \in I^{E}$, we set $A_{\mu, x^{\prime}}=\left\{\theta \in(0,1]: x^{\prime} \in[\mu]_{1-\theta}^{\circ}\right\}$. For $\mu \neq 0$, we define the fuzzy set $\mu^{\circ}$ on $E^{\prime}$ as

$$
\mu^{\circ}\left(x^{\prime}\right)= \begin{cases}\sup A_{\mu, x^{\prime}} & , \\ 0, & A_{\mu, x^{\prime}} \neq \varnothing, \\ A_{\mu, x^{\prime}}=\varnothing,\end{cases}
$$

and call it the fuzzy polar of $\mu$ in $E$.

Remark 1 ([3]). We note that if $\mu=1$, then for $\theta \in(0,1]$ we have $[\mu]_{1-\theta}=E$. Therefore $[\mu]_{1-\theta}^{\circ}=E^{\circ}=\{0\}$. This shows that for $x^{\prime} \neq 0, A_{\mu, x^{\prime}}=\varnothing$. Then $\mu^{\circ}\left(x^{\prime}\right)=0$ but clearly we have $\mu^{\circ}(0)=1$.

Lemma 2 ([3]). Let $\left(E, E^{\prime}\right)$ be a dual pair and $\mu \in I^{E}$. Then we have

$$
\mu^{\circ}=\sup _{\theta \in(0,1]} \theta \wedge \chi_{[\mu]_{1-\theta}^{\circ}} .
$$

Theorem 1 ([3]). If $\left(E, \tau_{f}\right)$ is a Hausdorff locally convex fuzzy topological vector space and $\mu$ is a fuzzy neighborhood of origin, then $\mu^{\circ}$ is $\sigma_{f}\left(E^{\prime}, E\right)$-compact.

Definition 11. A fuzzy subset $\mu$ in locally convex fuzzy topological vector space $E$ is said to be a fuzzy barrel if it is absolutely convex, absorbent and fuzzy closed.

In every Katsaras normed space $(E, \rho)$, the fuzzy set $\bar{\rho}$ (the closure of $\rho$ ) is absolutely convex, absorbent and fuzzy closed. Therefore $\bar{\rho}$ is a fuzzy barrel.

Proposition 3. Let $\left(E, \tau_{f}\right)$ be a locally convex fuzzy topological vector space. Then there is a linear fuzzy topology $\tau_{\mathfrak{B}}$ on $E$ such that all the fuzzy barrels of $\left(E, \tau_{f}\right)$ are fuzzy neighborhoods in $\left(E, \tau_{\mathfrak{B}}\right)$.

Proof. Let $\left(E, \tau_{f}\right)$ be a locally convex fuzzy topological vector space and $B$ be a fuzzy barrel in $E$. Then $B$ is a Katsaras seminorm in $E$ and the collection

$$
U_{B}=\{\theta \wedge(t B): t>0,0<\theta \leq 1\}
$$

is a neighborhood base for a fuzzy linear topology on $E$, denoted by $\tau_{B}$. Now, we set

$$
\tau_{\mathfrak{B}}=\bigcap_{\text {Bis a fuzzy barrel }} \tau_{B}
$$

The fuzzy topology $\tau_{\mathfrak{B}}$ is a linear fuzzy topology on $E$, since for each fuzzy barrel $B, \tau_{B}$ is a linear fuzzy topology. Also, it is clear that all the barrels are fuzzy neighborhoods in $\left(E, \tau_{\mathfrak{B}}\right)$.

It is clear that for the locally convex fuzzy topological vector space $\left(E, \tau_{f}\right)$ the fuzzy topology $\tau_{\mathfrak{B}}$ is weaker than $\tau_{f}$, since for each absolutely convex absorbent fuzzy neighborhood $\mu$ in $\tau_{f}$, the closure of $\mu$ is a fuzzy barrel. But the converse is not true in general. 
Definition 12. A locally convex fuzzy topological vector space $\left(E, \tau_{f}\right)$ is said to be a fuzzy barrelled space if every fuzzy barrel in $E$ is a fuzzy neighborhood. In other words, for each fuzzy barrel $\mu$ in $E$ there is a fuzzy neighborhood $\nu$ such that $\nu \leq \mu$.

Corollary 1. Let $\left(E, \tau_{f}\right)$ be a fuzzy barrelled locally convex space. Then the fuzzy topologies $\tau_{f}$ and $\tau_{\mathfrak{B}}$ are equivalent, since in the fuzzy barrelled spaces every fuzzy barrel is a fuzzy neighborhood. In fact for each neighborhood B in $\tau_{\mathfrak{B}}$ there is a fuzzy neighborhood $\mathrm{v}$ in $\tau_{f}$ such that $\mathrm{v} \leq B$.

Lemma 3. Let $(E, \tau)$ be a locally convex topological vector space, and $B$ be a barrel in $E$. Then $\chi_{B}$ is a fuzzy barrel in fuzzy topological vector space $(E, \omega(\tau))$.

Proof. Since $B$ is a barrel, then it is absolutely convex and closed. Now Proposition 2 shows that $\chi_{B}$ is fuzzy absolutely convex and closed. Also since $B$ is absorbing then for each $x \in B$ there is $\lambda>0$ such that $x \in \lambda B$. Then $\frac{x}{\lambda} \in B$. This shows that $\chi_{B}\left(\frac{x}{\lambda}\right)=1$. Then $\sup _{t>0}\left(t \chi_{B}\right)(x)=\sup _{t>0} \chi_{B}\left(\frac{x}{t}\right)=1$. So $\chi_{B}$ is absorbing.

Proposition 4. Let E be a Hausdorff locally convex fuzzy topological vector space with dual $E^{\prime}$. Then the fuzzy subset $\mu$ of $E$ is a fuzzy barrel if and only if $\mu$ is the fuzzy polar of a $\sigma_{f}\left(E^{\prime}, E\right)$-bounded fuzzy subset of $E^{\prime}$.

Proof. Let $\mu$ be a fuzzy polar of a $\sigma_{f}\left(E^{\prime}, E\right)$-bounded fuzzy subset of $E^{\prime}$. We prove that the fuzzy subset $\mu$ of $E$ is a fuzzy barrel. Firstly, we prove that $\mu^{\circ}$ is balanced. So it is enough to show that $\mu^{\circ}\left(t x^{\prime}\right) \geq \mu^{\circ}\left(x^{\prime}\right)$ for all $x^{\prime} \in E$ and $t \in \mathbb{R}$ with $|t| \leq 1$. We have

$$
\begin{aligned}
\mu^{\circ}\left(t x^{\prime}\right) & =\sup A_{\mu, t x^{\prime}}=\sup \left\{\theta \in(0,1]: t x^{\prime} \in[\mu]_{1-\theta}^{\circ}\right\} \\
& =\sup \left\{\theta \in(0,1]: x^{\prime} \in\left(t[\mu]_{1-\theta}\right)^{\circ}\right\}=\sup \left\{\theta \in(0,1]: x^{\prime} \in[t \mu]_{1-\theta}^{\circ}\right\} \\
& =\sup A_{t \mu, x^{\prime}}=(t \mu)^{\circ}\left(x^{\prime}\right)=\frac{1}{|t|} \mu^{\circ}\left(x^{\prime}\right) \\
& \geq 1 \times \mu^{\circ}\left(x^{\prime}\right)=\mu^{\circ}\left(x^{\prime}\right) .
\end{aligned}
$$

The convexity of $\mu^{\circ}$ is obvious, since $[\mu]_{1-\theta}^{\circ}$ is a convex set for each $\theta \in(0,1]$. We note that for each $x \in E ; \varphi_{x}\left(x^{\prime}\right)=<x, x^{\prime}>$ is a $\sigma_{f}\left(E^{\prime}, E\right)$-continuous linear functional on $E^{\prime}$. Now, since $\left(\theta \wedge \chi_{[\mu]_{1-\theta}^{\circ}}\right)$ is a closed subset of $\mathbb{K}$ and $\mu^{\circ}=\bigwedge_{\mu(x)>\theta} \varphi_{x}^{-1}\left(\theta \wedge \chi_{[\mu]_{1-\theta}^{\circ}}\right)$ then $\mu^{\circ}$ is $\sigma_{f}\left(E^{\prime}, E\right)$-closed. Conversely, suppose $\mu$ is a fuzzy barrel. Then it is absolutely convex and absorbent. Now, we prove that $\mu^{\circ}$ is $\sigma_{f}\left(E^{\prime}, E\right)-$ bounded. We have

$$
\mu^{\circ \circ}(x)=\sup \left\{\theta: x \in\left[\mu^{\circ}\right]_{1-\theta}^{\circ}\right\} .
$$

Let $\tau_{f}=w(\tau)$. Since $\mu$ is fuzzy closed and absolutely convex, so $[\mu]_{\theta}$ is closed and absolutely convex with respect to $\tau$. Then, we have

$$
\left[\mu^{\circ}\right]_{1-\theta}^{\circ}=\left([\mu]_{\theta}^{\circ}\right)^{\circ}=[\mu]_{\theta}^{\circ \circ}=[\mu]_{\theta} .
$$


This shows that

$$
\begin{aligned}
\mu^{\circ \circ}(x) & =\sup \left\{\theta: x \in\left[\mu^{\circ}\right]_{1-\theta}^{\circ}\right\} \\
& =\sup \left\{\theta: x \in[\mu]_{\theta}^{\circ \circ}\right\} \\
& =\sup \left\{\theta: x \in[\mu]_{\theta}\right\} \\
& =\mu(x) .
\end{aligned}
$$

The fuzzy set $\mu^{\circ}$ is $\sigma_{f}\left(E^{\prime}, E\right)$-compact by Theorem 1 . Hence $\mu^{\circ}$ is $\sigma_{f}\left(E^{\prime}, E\right)$-bounded.

Lemma 4. Let $\left(E, \tau_{f}\right)$ be a locally convex fuzzy topological vector space. Then fuzzy barrel absorbs every convex compact fuzzy set.

Proof. Let $\mu$ be a fuzzy barrel and $v$ be a convex compact fuzzy set. By Proposition 1 there is a linear locally convex topology $\tau$ on $E$ such that $\tau_{f}=\omega(\tau)$. Now, since $v$ is a compact fuzzy set, $[v]_{\theta}$ is compact in $(E, \tau)$ for each $0<\theta \leq 1$ by Proposition 2. Also for each $0<\theta \leq 1,[\mu]_{\theta}$ is a usual barrel. Then there is $\lambda>0$ such that $[v]_{\theta} \subseteq \lambda[\mu]_{\theta}$ for all $0<\theta \leq 1$. So if $v(x) \geq \theta$, then $\mu\left(\frac{x}{\lambda}\right) \geq \theta$. That is, $(\lambda \mu)(x) \geq \theta$. Thus $v \leq \lambda \mu$.

Theorem 2. The same fuzzy sets are bounded in every linear fuzzy topology of dual pair.

Proof. Let $\tau_{f}$ be any fuzzy topology of the dual pair $\left(E, E^{\prime}\right)$. Then $\tau_{f}$ is obviously finer than $\sigma_{f}\left(E, E^{\prime}\right)$. Then $\tau_{f}$-bounded fuzzy sets are $\sigma_{f}\left(E, E^{\prime}\right)$-bounded. Conversely, let $v$ be a $\sigma_{f}\left(E, E^{\prime}\right)$-bounded fuzzy set and $\mu$ be a closed absolutely convex $\tau_{f}$-neighborhood. Then $\nu^{\circ}$ be a fuzzy neighborhood in $E^{\prime}$ under $\sigma_{f}\left(E^{\prime}, E\right)$ and $\mu$ is absolutely convex and $\sigma_{f}\left(E^{\prime}, E\right)$-compact by Theorem 1 . Hence by Lemma $4, v^{\circ}$ absorbs $\mu^{\circ}$. Then $\mu^{\circ \circ}$ absorbs $v^{\circ \circ}$. Since $\mu \leq \mu^{\circ \circ}$ and $v^{\circ \circ}=v$. Then $\nu$ absorbs $\mu$. This shows that $v$ is $\tau_{f}$-bounded.

Definition 13. Let $\left(E, \tau_{f}\right)$ be a fuzzy topological vector space. The family $\mathcal{F} \subseteq E^{\prime}$ is called fuzzy equicontinuous if for each fuzzy neighborhood $\mu$ in $\mathbb{K}$, there is a fuzzy neighborhood $v \in \tau_{f}$ such that $f(v) \leq \mu$ for all $f \in \mathcal{F}$.

Corollary 2. Let $E$ be Hausdorff locally convex fuzzy topological vector space with dual $E^{\prime}$ then $E$ is fuzzy barrelled if and only if every $\sigma_{f}\left(E^{\prime}, E\right)$-bounded fuzzy subset of $E^{\prime}$ is fuzzy equicontinuous.

Corollary 3. Let $E$ be Hausdorff locally convex fuzzy topological vector space with dual $E^{\prime}$, then $E$ has the fuzzy topology $\tau_{f}\left(E, E^{\prime}\right)$.

Definition 14. Let $E$ and $F$ be locally convex fuzzy topological vector spaces and $P$ be a collection of linear mappings from $E$ into $F$. We say that $P$ is pointwise 
bounded if for every $x \in E$ and every absolutely convex closed fuzzy neighborhood $v$ in $F$ we have

$$
\bigvee_{t>0} \bigwedge_{T \in P}(t \vee)(T(x))=1
$$

One of the powerful theorems of functional analysis, the Banach-Steinhaus theorem, asserts that the set of continuous linear mapping that is bounded at each point of a Banach space is bounded uniformly on the unit ball. In the following theorem we prove the Banach-Steinhaus theorem for fuzzy barrelled spaces.

Theorem 3. Let $E$ be a fuzzy barrelled space and $F$ a fuzzy convex space. Then any pointwise bounded fuzzy set of fuzzy continuous linear mappings of $E$ into $F$ is fuzzy equicontinuous.

Proof. Let $P$ be a pointwise fuzzy bounded set of continuous linear operators from $E$ into $F$. Suppose $v$ is a fuzzy closed absolutely convex fuzzy neighborhood in $F$. We set $B=\bigwedge_{T \in P} T^{-1}(v)$. Then $B$ absolutely convex, and closed. We prove that $B$ is also absorbing. Since $v$ is absorbing for $x \in E$ we have

$$
\begin{aligned}
\sup _{t>0}(t B)(x) & =\bigvee_{t>0} \bigwedge_{T \in P} T^{-1}(\mathrm{v})\left(\frac{x}{t}\right) \\
& =\bigvee_{t>0} \bigwedge_{T \in P} v\left(T\left(\frac{x}{t}\right)\right) \\
& =\bigvee_{T \in P} \bigwedge_{t>0} v\left(\frac{T(x)}{t}\right) \\
& =1
\end{aligned}
$$

Hence $B$ is a fuzzy barrel in $E$. Now, since $E$ is barrelled, then $B$ is a fuzzy neighborhood. Also for each $T^{\prime} \in P$ we have

$$
\begin{aligned}
T^{\prime}(B)(y) & \leqslant T^{\prime}\left(\left(T^{\prime}\right)^{-1}(\mathrm{v})\right)(y) \\
& =\sup _{x \in\left(T^{\prime}\right)^{-1}(y)}\left(\left(T^{\prime}\right)^{-1}(\mathrm{v})\right)(x) \\
& =\sup _{x \in\left(T^{\prime}\right)^{-1}(y)} v\left(T^{\prime}(x)\right) \\
& \leqslant \mathrm{v}(y) .
\end{aligned}
$$

This shows that $P$ is equicontinuous.

Example 1. Consider the fuzzy locally convex space $\mathbb{R}^{n}$ endowed with the fuzzy Katsaras norm

$$
\rho\left(x_{1}, \ldots x_{n}\right)= \begin{cases}1 & \Sigma_{i=1}^{n} x_{i}^{2}<1 \\ 0 & \text { else }\end{cases}
$$


It is clear that $\mathbb{R}^{n}$ is complete (a fuzzy Banach space). We prove that $\mathbb{R}^{n}$ is barrelled. We have

$$
\bar{\rho}\left(x_{1}, \ldots . x_{n}\right)= \begin{cases}1 & \sum_{i=1}^{n} x_{i}^{2} \leq 1, \\ 0 & \text { else. }\end{cases}
$$

This show that $\bar{\rho}$ is compact (since $\mathbb{R}^{n}$ is complete). Now, suppose $B$ is a fuzzy barrel in $\mathbb{R}^{n}$. By Lemma $4, B$ absorbs $\bar{\rho}$ i.e. for each $0<\theta \leq B(0)$ there is $t>0$ such that $\theta \wedge(t \bar{\rho}) \leq B$. This yields that $\theta \wedge(t \rho) \leq \theta \wedge(t \bar{\rho}) \leq B$. Then $B$ absorbs $\rho$. Thus $B$ is a fuzzy neighborhood in $\mathbb{R}^{n}$ and $\mathbb{R}^{n}$ is barrelled.

Example 2. Consider the fuzzy locally convex spaces $E=\mathbb{R}^{n}$ and $F=\mathbb{R}$ endowed with the Katsaras fuzzy norms

$$
\rho\left(x_{1}, \ldots x_{n}\right)= \begin{cases}1 & \Sigma_{i=1}^{n} x_{i}^{2}<1 \\ 0 & \text { else }\end{cases}
$$

and

$$
v(x)= \begin{cases}1 & |x|<1, \\ 0 & \text { else } .\end{cases}
$$

For $i=1,2, \cdots, n$ let $T_{i}\left(x_{1}, x_{2}, \cdots, x_{n}\right)=x_{i}$. Then the collection $P=\left\{T_{i}: i=1,2\right.$, $\cdots, n\}$ is pointwise bounded since

$$
\begin{aligned}
\bigvee_{t>0} \bigwedge_{i=1, \cdots, n}(t v)\left(T_{i}\left(x_{1}, \cdots, x_{n}\right)\right) & =\bigvee_{t>0} \bigwedge_{i=1, \cdots, n}(t v)\left(x_{i}\right) \\
& =\bigvee_{t>0} \bigwedge_{i=1, \cdots, n} v\left(\frac{x_{i}}{t}\right) \\
& =v(0) \\
& =1
\end{aligned}
$$

Now, since $E$ is fuzzy barrelled, by Theorem 3, $P$ is fuzzy equicontinuous.

\section{CONClusion}

The Banach-Steinhaus theorem is one of the important theorems in functional analysis, which asserts that the set of continuous linear mapping that is pointwise bounded is bounded uniformly on barrelled spaces. Theorem 3 is an extension of Banach-Steinhaus theorem to the more general case of fuzzy locally convex spaces.

\section{ACKNOWLEDGMENT}

The authors are grateful to the referees for their valuable comments and suggestions, which have greatly improved the paper. 


\section{REFERENCES}

[1] T. Bag and S. K. Samanta, "Finite dimensional fuzzy normed linear spaces." The Journal of Fuzzy Mathematics., vol. 11, no. 3, pp. 687-705, 2003.

[2] S. C. Cheng and J. N. Mordeson, "Fuzzy linear operators and fuzzy normed linear spaces." Bulletin of the Calcutta Mathematical Society., vol. 86, no. 5, pp. 429-436, 1994.

[3] B. Daraby, N. Khosravi, and A. Rahimi, "Polar fuzzy topologies on fuzzy topological vector spaces." arXiv preprint arXiv:1910.04152., 2019.

[4] B. Daraby, N. Khosravi, and A. Rahimi, "Weak fuzzy topology on fuzzy topological vector spaces.” arXiv preprint arXiv:1910.03405., 2019.

[5] B. Daraby, Z. Solimani, and A. Rahimi, "A note on fuzzy Hilbert spaces.” Journal of Intelligent \& Fuzzy Systems., vol. 31, pp. 313-319, 2016, doi: 10.3233/IFS-162143.

[6] B. Daraby, Z. Solimani, and A. Rahimi, "Some properties of fuzzy Hilbert spaces." Complex Analysis and Operator Theory., vol. 11, pp. 119-138, 2017, doi: 10.1007/s11785-016-0581-0.

[7] N. R. Das and P. Das, "Fuzzy topology generated by fuzzy norm." Fuzzy Sets and Systems., vol. 107, pp. 349-354, 1999, doi: 10.1016/S0165-0114(97)00302-3.

[8] J. X. Fang, "On local bases of fuzzy topological vector spaces." Fuzzy Sets and Systems., vol. 87, pp. 341-347, 1997, doi: 10.1016/0165-0114(95)00364-9.

[9] J. X. Fang, "On I-topology generated by fuzzy norm." Fuzzy Sets and Systems., vol. 157, pp. 2739-2750, 2006, doi: 10.1016/j.fss.2006.03.024.

[10] C. Felbin, "Finite dimensional fuzzy normed linear space." Fuzzy Sets and Systems., vol. 48, pp. 239-248, 1992, doi: 10.1016/0165-0114(92)90338-5.

[11] A. K. Katsaras, "Fuzzy topological vector spaces I." Fuzzy Sets and Systems., vol. 6, pp. 85-95, 1981, doi: 10.1016/0165-0114(81)90082-8.

[12] A. K. Katsaras, "Fuzzy topological vector spaces II." Fuzzy Sets and Systems., vol. 12, no. 2, pp. 143-154, 1984, doi: 10.1016/0165-0114(84)90034-4.

[13] A. K. Katsaras and D. B. Liu, "Fuzzy vector spaces and fuzzy topological vector spaces." Journal of Mathematical Analysis and Applications., vol. 58, pp. 135-146, 1977, doi: 10.1016/0022247X(77)90233-5.

[14] A. P. Robertson and W. Robertson, Topological vector spaces, Cambridge University Press, 1980.

[15] R. H. Warren, "Neighborhoods bases and continuity in fuzzy topological spaces." Rocky Mountain Journal of Mathematics., vol. 8, pp. 459-470, 1978.

[16] J. Xiao and X. Zhu, "On linearly topological structure and property of fuzzy normed linear space." Fuzzy Sets and Systems., vol. 125, pp. 153-161, 2002, doi: 10.1016/S0165-0114(00)00136-6.

Authors' addresses

B. Daraby

Department of Mathematics, University of Maragheh, P. O. Box 55136-553, Maragheh, Iran

E-mail address: bdarabyemaragheh.ac.ir

N. Khosravi

Department of Mathematics, University of Maragheh, P. O. Box 55136-553, Maragheh, Iran

E-mail address: nasibeh_khosravi@yahoo.com

A. Rahimi

Department of Mathematics, University of Maragheh, P. O. Box 55136-553, Maragheh, Iran

E-mail address: rahimi@maragheh.ac.ir 